\title{
Cirugía bariátrica en la morbimortalidad de la infección por SARS-CoV-2
}

\section{Bariatric surgery on the morbidity and mortality of SARS-CoV-2 infection}

$\mathrm{DOI}$

https://doi.org/10.35434/rcmhnaaa.2021.143.1277

\section{RESUMEN}

Introducción: La pandemia COVID-19 ha tenido un gran impacto en todo el mundo y al ser la obesidad es un factor de riesgo importante para la forma grave de la enfermedad por SARS-CoV-2 la cirugía bariátrica tendría gran impacto clínico. La realización de esta cirugía en personas con obesidad, implica una pérdida de peso, la cual tiene múltiples efectos beneficiosos como reducir la respuesta inflamatoria, incrementar la inmunidad y mejorar la función cardiopulmonar y renal. Esta revisión tiene el propósito de proveer información actualizada sobre la cirugía bariátrica en la morbimortalidad de la infección por SARS-CoV-2. Se espera que brinde apoyo al lector a fin de entender los mecanismos fisiopatológicos implicados en el mejor pronóstico y menor probabilidad de hospitalización y mortalidad por COVID-19, en comparación de los pacientes obesos que no han sido sometidos a cirugía bariátrica.

Palabras Clave: Obesidad, COVID-19, Cirugía Bariátrica (Fuente: DeCS-BIREME).

\section{ABSTRACT}

Background: The COVID-19 pandemic has had a big impact around the world and as obesity is an important risk factor for the severe form of the SARS-CoV-2 disease, bariatric surgery will have a great clinical impact. Performing this surgery in people with obesity implies weight loss, which has multiple beneficial effects such as reducing the inflammatory response, increasing immunity and improving cardiopulmonary and kidney function. This review is intended to provide updated information on bariatric surgery in the morbidity and mortality of SARS-CoV-2 infection. Expecting that it provides support to the reader in order to understand the pathophysiological mechanisms involved in the better prognosis and lower probability of hospitalization and mortality from COVID-19, pending comparison of obese patients who have not undergone bariatric surgery.

Keywords: Obesity, COVID-19, Bariatric Surgery. (Source: DeCSBIREME).

\section{FILIACIÓN}

1. Facultad de Medicina, Universidad Nacional de Trujillo, Trujillo, Perú.

2. Departamento de Fisiología, Facultad de Medicina. Universidad Nacional de Trujillo, Trujillo, Perú.

a. Estudiante de Medicina.

b. Doctor en Ciencias Biomédicas.

ORCID

1. Ayumi Castillo-de la Cruz / 0000-0003-3972-4957

2. Alexia Carrión-Arias / 0000-0003-1909-0926

3. Julio Hilario-Vargas / 0000-0002-8656-6843

CORRESPONDENCIA

Julio Hilario-Vargas

EMAIL

jhilario@unitru.edu.pe

CONFLICTOS DE INTERÉS

Los autores niegan conflictos de interés.

FINANCIAMIENTO

Autofinanciamiento.

REVISIÓN DE PARES

Recibido: $12 / 05 / 2021$

Aceptado: 09/09/2021

\section{COMO CITAR}

Castillo-de la Cruz, A., Carrión-Arias, A., \& Hilario-Vargas, $J$. Cirugía bariátrica en la morbimortalidad de la infección por SARS-CoV-2. Revista Del Cuerpo Médico Hospital Nacional Almanzor Aguinaga Asenjo, 2021, 14(3), 367 - 374. https://doi.org/10.35434/rcmhnaaa.2021.143.1277 


\section{INTRODUCCIÓN}

La obesidad se define como "una acumulación anormal o excesiva de grasa que puede ser perjudicial para la salud", según la $\mathrm{OMS}^{(1)}$. Es una condición que se mide por la relación entre el peso y la talla al cuadrado $(I M C)^{(2)}$, el cual debe ser mayor o igual a $30^{(1)}$.

En el Perú, según los datos proporcionados por el INS (Instituto Nacional de Salud) en el año 2020, de las personas mayores de 15 años, el $37,9 \%$ padece de sobrepeso y el $24,6 \%$ de obesidad $^{(3)}$.

Los pacientes con obesidad suelen presentar comorbilidades como: diabetes mellitus, síndrome metabólico, resistencia a la insulina, enfermedad cardiovascular, enfermedad renal; además de una disfunción respiratoria, que es caracterizada por alteraciones en los mecanismos respiratorios, aumento de la resistencia de las vías respiratorias, alteración del intercambio de gases y bajo volumen pulmonar y fuerza muscular. Por lo cual, se considera a estos individuos más vulnerables a las fallas orgánicas asociadas a la neumonía producida en el cuadro grave por COVID-19 ${ }^{(4)}$.

Una forma eficaz para el control de la enfermedad del COVID19 es el control de sus factores de riesgo ${ }^{(5)}$; entre estos, se encuentra la obesidad mórbida, un factor de mal pronóstico en la infección por SARS-COV- $2^{(5,6)}$. Uno de los tratamientos más eficaces para la obesidad es la cirugía bariátrica, la cual ayuda en la disminución del riesgo de resultados deficientes de salud física y mental relacionados con COVID-19 y la obesidad $^{(6)}$. Hay varios tipos de cirugía bariátrica a realizar; sin embargo, el perfil más favorable en beneficio, como el mayor porcentaje de pérdida de peso y menos complicaciones quirúrgicas, es la cirugía bariátrica de bypass gástrico en $Y$ de Roux (BGYR) ${ }^{(7)}$; siendo el procedimiento quirúrgico bariátrico con una mayor proporción a corto plazo de exceso de pérdida de peso ${ }^{(8)}$.

\section{OBESIDAD COMO FACTOR DE RIESGO PARA LA INFECCIÓN POR SARS-COV-2}

La obesidad puede provocar grandes cambios en el funcionamiento del organismo, lo cual la hace más vulnerable al individuo frente al riesgo de complicación por COVID-19(figura 1). Entre estos cambios tenemos ${ }^{(9)}$ :

- La obesidad afecta la inmunidad al alterar la respuesta de las citocinas, esto conduce a la disminución en la respuesta de las células citotóxicas que tienen un papel importante en la defensa de nuestro cuerpo frente a infecciones virales ${ }^{(9)}$.

Perturba el equilibrio de las hormonas endocrinas. El tejido adiposo produce leptina, una hormona que regula el apetito a nivel del SNC, lo cual afecta la interacción entre las células metabólicas y el sistema inmunológico ${ }^{(9)}$.

Conduce al aumento de la producción y la participación de adipocinas por las células dendríticas; lo cual genera un entorno favorable para enfermedades inmunológicas. Esto se observa en la mayor producción de IL 10 (citocina inmunosupresora) y de IL 4 (células T alogénicas) ${ }^{(9)}$.
Disminuye la capacidad de las células dendríticas para madurar y provocar respuestas apropiadas de células $T$ a un estímulo por infección viral ${ }^{(9)}$.

El nivel de expresión de los receptores ACE2 en el tejido adiposo es más alto que en el tejido pulmonar ${ }^{(9)}$. Estos receptores tienen una relación directa con la masa de los tejidos adiposos, lo cual hace que los pacientes con obesidad expresan un elevado número de receptores. Este incremento podría explicar el porqué los pacientes con obesidad presentan una forma grave de Covid-19(9).

- $\quad$ Es fundamental tener en cuenta que los pacientes con obesidad también padecen de otros trastornos metabólicos como la diabetes, hipertensión, enfermedades cardíacas y cerebrovasculares, lo cual no debe ignorarse porque el riesgo acumulativo de mortalidad aumenta con las comorbilidades asociadas $^{(9)}$.

- Tiene un efecto negativo sobre los volúmenes, funciones y expansiones pulmonares ${ }^{(9)}$.

Todo esto conduce al mal pronóstico de los pacientes obesos con COVID 19, lo cual se evidencia en las cifras reportadas $^{(4)}$.

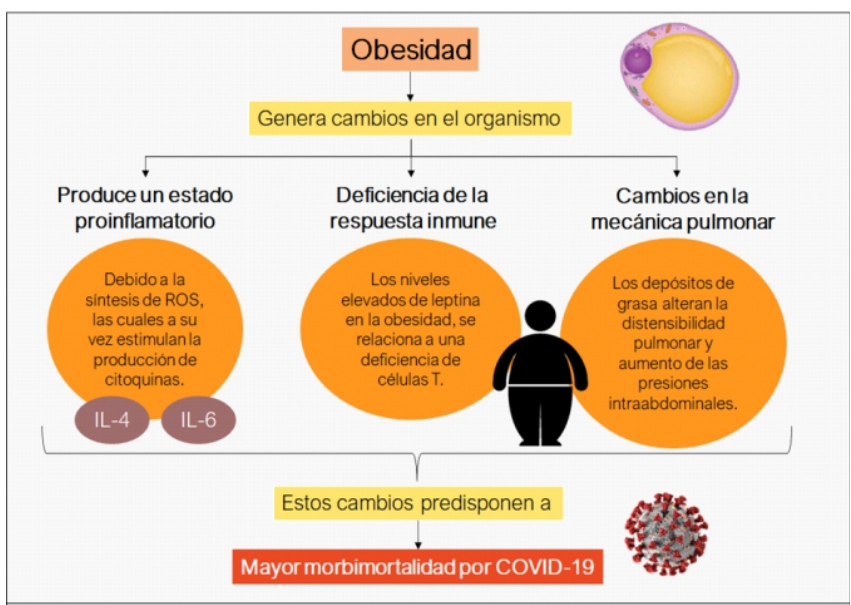

Figura 1.

Obesidad como factor de riesgo para la infección por SARS-CoV-2.

\section{Estado proinflamatorio}

En la obesidad, la presencia de tejido adiposo excesivo, constituye una fuente de citocinas proinflamatorias, lo cual incluye el factor de necrosis tumoral alfa (TNF- $\alpha$ ), la interleucina (IL) -1B e IL-6; las cuales promueven una mayor generación de ROS (Especies reactivas del oxígeno) y nitrógeno por macrófagos y monocitos; por lo tanto, un aumento de la concentración podría ser responsable de un aumento del estrés oxidativo ${ }^{(10,11)}$.

El TNF-a es una citocina crítica que influye en procesos como el metabolismo de los lípidos, lo cual aumenta la lipogénesis hepática, la señalización de la insulina y la inducción del estrés oxidativo. La producción de ROS también puede ser inducida por TNF-a mediante la unión de receptores específicos y la promoción de la señalización de NF-KB (Factor nuclear kappa B). TNF-a conduce al reclutamiento de TRAF2 (Factor 2 asociado al receptor de TNF) a la membrana plasmática, luego de unirse a su receptor TRAF2, que actúa 
como regulador central de las vías de transducción de señales pro y antiapoptóticas. Esta interacción desencadena la unión a proteínas, como A20, luego se activan las quinasas y factores de transcripción, como NIK (Quinasa inductora del $N F-k B)$ y NF-B ${ }^{(10)}$.

Los ROS inducen la liberación adicional de citocinas proinflamatorias y la expresión de moléculas de adhesión y factores de crecimiento a través de factores de transcripción sensibles a redox, en particular NF-kB y la vía NADPH oxidasa (NOX), de las cuales, NOX4 corresponde a los adipocitos. Asimismo, algunas ROS se convierten en peróxido de hidrógeno, el cual también estimula la expresión génica de IL-4 e IL-6 y la secreción de citocinas ${ }^{(11)}$.

Además, el tejido adiposo blanco se modifica patológicamente. Cuando este tejido alcanza su máxima expansión, se inhibe la angiogénesis, generando hipoxia crónica y procesos fibróticos en el tejido. El tejido adiposo envejece prematuramente, caracterizándose por su disfunción mitocondrial, senescencia celular, acortamiento de los telómeros y un fenotipo secretor asociado a la senescencia. Todos estos cambios conducen a la creación de un medio proinflamatorio local que induce resistencia a la insulina en los adipocitos, que en el transcurso del tejido adiposo se disemina a todo el organismo ${ }^{(11,12)}$.

\section{Deficiencia de la respuesta inmune}

La obesidad genera cambios en el sistema inmunológico ${ }^{(13)}$ :

- $\quad$ Afecta la inmunidad humoral, a través de los cambios en la liberación de anticuerpos ${ }^{(13)}$.

- $\quad$ Afecta la inmunidad celular, a través de los cambios en el recuento de leucocitos y las subpoblaciones de linfocitos ${ }^{(13) .}$

- Una de las funciones del tejido adiposo es la endocrina, que consiste en la producción de hormonas y otras moléculas de señalización celular, como la leptina, la cual está implicada en el equilibrio energético y en la regulación de la respuesta inmunológica ${ }^{(1,13)}$.

Uno de los efectos de la leptina es la estimulación de células inmunológicas como los monocitos y linfocitos $T$ para que aumenten sus receptores celulares y de esa manera, al activar estas células, se produce la liberación de moléculas proinflamatorias como IL-2 e IFN-y. Por esta razón, se considera a la leptina como una citocina proinflamatoria ${ }^{(13)}$. Sin embargo, los niveles elevados de leptina en la obesidad, provocan la desensibilización de sus receptores celulares y originan de está manera una resistencia a ella misma, lo que ocasiona que las células T perciban una deficiencia de leptina y no se activen adecuadamente durante la respuesta inmunológica. Esto se asocia con la mayor incidencia de infecciones en pacientes obesos ${ }^{(13,14)}$.

\section{Cambios en la mecánica pulmonar}

La coexistencia de un estado proinflamatorio, con altos niveles de citocinas y estrés oxidativo por exceso de grasa, deficiencias en la respuesta inmune, cambios restrictivos en la mecánica de los pulmones y la pared torácica y respuesta limitada a la ventilación mecánica pueden contribuir a un mal pronóstico en pacientes con COVID-19 con obesidad ${ }^{(15,16)}$.

El impacto de la obesidad en la función pulmonar es multifactorial, donde se destaca principalmente los aspectos mecánicos e inflamatorios previamente explicados, contribuyen a los síntomas respiratorios asociados como disnea, sibilancias e hiperactividad en las vías respiratorias $^{(17,18)}$

Los depósitos de grasa en el mediastino y las cavidades abdominales, originan en gran parte la alteración de la mecánica pulmonar, desencadenando una menor distensibilidad pulmonar por aumento de su rigidez, y aumento de las presiones intraabdominales y pleurales ${ }^{(17,18)}$. Además, de generar un estrechamiento y cierre de las vías respiratorias, originando un atrapamiento del aire, la falta de homogeneidad de la ventilación; así como, un aumento de la resistencia del sistema respiratorio ${ }^{(17)}$.

Por ende, se altera el patrón respiratorio, llevando a una gran reducción del volumen de reserva espiratoria (VRE) y de la capacidad residual funcional (CRF); la disminución de esta última es proporcional a la gravedad de la obesidad ${ }^{(17)}$.

\section{Respuesta limitada de la ventilación pulmonar}

La obesidad tiene muy poco efecto sobre el volumen residual (RV) y la capacidad pulmonar total (TLC); pero, un gran efecto en la disminución significativa de la capacidad residual funcional (FRC) y el volumen de reserva espiratoria $(\mathrm{ERV})^{(17)}$.

Otras medidas dinámicas de la función pulmonar, como el FEV1 (Volumen espirado forzado en el 1 segundo) y la FVC (Capacidad vital forzada), se reducen ligeramente en presencia de obesidad, pero la relación FEV 1 / FVC se ve afectada generalmente cuando el IMC es superior a $62 \mathrm{~kg} /$ $\mathrm{m} 2^{(17)}$.

Por lo tanto, la mecánica respiratoria desventajosa en los sujetos obesos puede afectarse gravemente ante cualquier daño respiratorio, esto dificultará la recuperación de los pacientes que son afectados por el SARS-COV-2. Por lo tanto, estas personas son las más vulnerables ${ }^{(18)}$.

\section{DATOS RELACIONADOS CON PACIENTES OBESOS EN UCI}

Diversos estudios realizados en todo el mundo, ponen en evidencia la relación de la obesidad como factor de riesgo en la infección por COVID 19.

Una cohorte realizada en Francia, demostró una alta frecuencia de obesidad en pacientes ingresados a $\mathrm{UCI}$ (Unidad de Cuidados Intensivos) por SARS-CoV-2. Concluyendo que la gravedad de la enfermedad aumentó con el IMC ${ }^{(19)}$.

Un estudio en Nueva York realizado a pacientes COVID-19, determinó que existe una asociación significativa de la obesidad con la necesidad de hospitalización y el estado crítico de los pacientes en cuidados intensivos, ventilación mecánica y/o muerte ${ }^{(20)}$. Otro estudio en pacientes británicos confirma la asociación entre la obesidad y un riesgo elevado de muerte por COVID-19(21).

En China detectaron, en datos preliminares de COVID-19 que, entre los pacientes críticos fallecidos el $88,24 \%$ presentaban un $I M C>25 \mathrm{~kg} / \mathrm{m} 2$. En Europa, se reporta que un mayor 
número de personas con obesidad necesitan ingreso en $\mathrm{UCl}$ y hasta 7 veces más ventilación mecánica intensiva $(\mathrm{VMI})^{(22)}$.

Estos antecedentes sugieren un punto de corte de IMC ( $\geq 35$ $\mathrm{kg} / \mathrm{m} 2$ ) que condiciona mayor gravedad por COVID-19. Sin embargo, estudios recientes confirman que cualquier grado de obesidad confiere un incremento tanto del riesgo de gravedad como de mortalidad por COVID-19 $9^{(19-22)}$.

En el Perú, el reporte del Sistema Informático Nacional de Defunciones (Sinadef) indica que el $85.5 \%$ de los pacientes fallecidos por COVID-19 padecían de obesidad ${ }^{(23)}$.

\section{BENEFICIOS DE LA CIRUGÍA BARIÁTRICA EN LACOVID-19}

La cirugía bariátrica es una opción cada vez más popular en su tratamiento. Recientemente, la cirugía bariátrica ha sido reconocida como un importante modificador de los estados metabólicos y hormonales asociados con las comorbilidades de la obesidad ${ }^{(24)}$.

La cirugía bariátrica conduce a una pérdida de peso sustancial, sostenida y a un perfil de salud mejorado en general. La reducción del exceso de tejido adiposo reduce la respuesta inflamatoria, aumenta la inmunidad, mejora la función cardiopulmonar y renal y reduce el riesgo de eventos cardiovasculares mayores. Esta mejora en los factores de riesgo cardiometabólico probablemente explica la mejora de los resultados clínicos en pacientes con obesidad severa que dan positivo al SARS-CoV-2 ${ }^{(25)}$.

La cirugía bariátrica ha mostrado efectos favorables sobre las comorbilidades asociadas con la obesidad incluyendo la mejora de la función pulmonar, lo cual influye en la disminución de las tasas de hospitalización y mortalidad de COVID-19(25-27)

\section{Disminución de la morbimortalidad post cirugía:}

Ali Aminiam y colaboradores ${ }^{(26)}$, concluyeron que la reducción del IMC al realizar la cirugía bariátrica se asoció con una menor tasa de ingreso hospitalario, en comparación con aquellos que no se realizaron la cirugía. Estos últimos requirieron ventilación mecánica, diálisis e incluso algunos de ellos llegan a fallecer ${ }^{(26)}$.

Cabe resaltar que estos resultados fueron obtenidos de una cohorte retrospectiva, por lo que se deben considerar las limitaciones inherentes al diseño del estudio al estar expuesto a sesgos de información.

Este mismo autor realizó una revisión sistemática en la cual se reafirma el efecto benéfico de la cirugía bariátrica; donde el riesgo de mortalidad sin cirugía bariátrica previa fue de 133 por 1000 casos; en comparación con la tasa de mortalidad con cirugía bariátrica previa fue de 33 por 1000 casos. Así mismo, la tasa de hospitalización en pacientes sin cirugía bariátrica previa fue de 412 por 1000 casos y la tasa en pacientes con cirugía bariátrica previa fue de 164 por $1000^{(27)}$.

Marchesi pone en evidencia que pacientes sometidos a cirugía bariátrica desarrollan una infección por SARS-CoV-2 menos grave que los sujetos que padecen obesidad. También, una disminución en la incidencia de la sintomatología relacionada con COVID-19, como anosmia, disgeusia y fiebre de inicio rápido ${ }^{(25)}$.

Los estudios demuestran, luego de un análisis cuantitativo de los datos disponibles, que el $44,8 \%$ de los controles y el $18,2 \%$ de los pacientes de cirugía bariátrica ingresaron en el hospital con COVID-19. De estos, el $13 \%$ de los controles ingresaron en la $\mathrm{UCl}$, el 1,5\% necesitó diálisis, el 6,7\% requirió ventilación mecánica y el $2,4 \%$ falleció ${ }^{(28,29)}$.

Uccelli y colaboradores, realizaron un análisis retrospectivo en el 2020, en la cual se identificaron pacientes que habían sido sometidos a cirugía bariátrica y siguieron los casos para evaluar su cuadro en caso se contagien de COVID-19. Sus resultados evidencian que del total de 2145 pacientes, de los cuales el $8,4 \%$ contrajeron la enfermedad, de estos últimos, solo 2 personas necesitaron acudir a UCI. Por lo que podemos observar que el $0.1 \%$ del total de participantes desarrollaron complicaciones, las cuales les llevaron a entrar a sala de $\mathrm{UCl}^{(30)}$.

En un estudio de cohorte internacional elaborado por Singhal y colaboradores, identificaron de 2001 pacientes adultos que se sometieron a cirugía bariátrica y metabólica, sólo el $0,5 \%$ pacientes fueron diagnosticados con COVID-19 sintomático a los 30 días del postoperatorio; sin embargo, ninguno necesitó ventilación ni murió. Cabe resaltar que al ser una cohorte retrospectiva se deben considerar las limitaciones inherentes al diseño del estudio al estar expuesto a sesgos de información ${ }^{(31)}$.

La evaluación y preparación de los pacientes para la cirugía bariátrica no ha sido sustancialmente alterada, excepto por la detección de pacientes por una posible infección con el SARS-CoV- $2^{(16)}$.

Así mismo, al preparar al paciente para la cirugía bariátrica, es indispensable tener un correcto control de las enfermedades secundarias o relacionadas con la obesidad, como DM2 (Diabetes Mellitus tipo 2), HTA (Hipertensión arterial) o AOS (Apnea obstructiva del sueño), que pueden haber sido afectadas por el efecto del confinamiento durante la pandemia de COVID-19 $9^{(16)}$.

Sin embargo, se debe tener en cuenta los niveles altos de estrés originado en este tiempo de pandemia, lo cual puede exacerbar la impulsividad, desencadenando episodios de atracones antes y después de la cirugía bariátrica ${ }^{(16)}$.

\section{Riesgos:}

Por retraso en la cirugía bariátricas:

Se ha observado que el retraso en la cirugía bariátrica tiene consecuencias fatales: hasta el $50 \%$ de los pacientes desarrollan una nueva comorbilidad y el $1,5 \%$ mueren mientras están en la lista de espera ${ }^{(16)}$.

Es importante atender cuanto antes a pacientes que estén considerando la cirugía bariátrica, debido a los riesgos de complicaciones graves por COVID-19 en pacientes con obesidad y diabetes tipo 2; además, de que las personas con obesidad también son propensas a enfermedades cardiovasculares, insuficiencia cardiaca, síndrome de hipoventilación, y otras afecciones que podrían aumentar el riesgo de mortalidad por COVID-19 ${ }^{(32)}$. 
Relacionados con el procedimiento:

Procedimientos donde se hace el uso de aerosoles como dióxido de carbono, neumoperitoneo, electrocauterio y cizallamiento ultrasónico. Por ejemplo, la cirugía laparoscópica o la endoscopia gastrointestinal superior realizada antes de la cirugía propiamente dicha, podrían aumentar el riesgo de contagio viral por SARS-COV-2 para el personal $^{(32)}$.

Por esto lo mejor es posponer la cirugía bariátrica durante la fase I (infección temprana) de la persona infectada por COVID-19, a excepción de la cirugía de revisión urgente o emergente como en obstrucción, hemorragia, banda gástrica deslizada, hernia interna, perforación o hemorragia de úlcera marginal ${ }^{(16,32)}$. En las cuales se deberá hacer el uso adecuado de equipo de protección, dado el mayor riesgo de infección por SARS-CoV-2 ${ }^{(32)}$.

\section{Fisiopatología de la cirugía bariátrica en COVID-19 (Figura} 2).

\section{Pérdida de peso}

Al inicio, la cirugía bariátrica consideraba dos mecanismos principales para la pérdida de peso postquirúrgico, la restricción de ingesta calórica y/o la mala absorción de nutrientes. Esta última traía riesgos en el paciente puesto que alteraba su flujo de nutrientes y sus enzimas digestivas. Sin embargo, el perfil favorable de riesgo-beneficio se vio reflejado en la cirugía bariátrica de $B G Y R^{(8)}$

La restricción y malabsorción postquirúrgica son conceptos simples que no explican la compleja fisiología de la obesidad ni los efectos metabólicos de la cirugía bariátrica ${ }^{(8)}$.

El ECA2, principal receptor implicado en la patología del SARS-COV-2, también se encuentra expresado en gran medida en el tejido adiposo y significativamente más en el tejido adiposo visceral que en el subcutáneo periférico ${ }^{(33,34)}$. Por ende, en los pacientes obesos el aumento de la expresión de la ECA 2 predispone a una exposición más masiva al virus y el desarrollo de una forma más severa de la enfermedad. Es por esto que las personas sometidas a cirugía bariátrica, que conlleva a la pérdida de tejido adiposo, tienen menor riesgo de gravedad de COVID-19 en comparación con las personas obesas sin cirugía bariátrica ${ }^{(34)}$.

\section{Efecto entero-hormonal}

El tracto gastrointestinal implica un gran sistema neuroendocrino con más de 100 hormonas, de las cuales destacan las incretinas, producidas en respuesta a un estímulo alimentario. La incretina mejor estudiada es el péptido similar al glucagón tipo 1 (GLP-1), el cual desempeña un papel importante después de la cirugía bariátrica ${ }^{(35)}$.

La cirugía de RYGB permite una llegada más rápida de los nutrientes al intestino posterior, originando un aumento más rápido de los niveles de GLP-1 ${ }^{(35)}$. Este aumento del GLP-1, suprime al glucagón y la grelina, hormonas implicadas en la hiperglucemia y un estado de apetito elevado, respectivamente. Contribuyendo de esta manera a una mejora de la hiperglucemia posprandial y menor ingesta calórica por la supresión del apetito ${ }^{(8,35)}$.

\section{- Resolución de la insulinoresistencia}

La pérdida de peso es un factor indispensable para aquellos pacientes con comorbilidades asociadas a su obesidad, como la Diabetes Mellitus. Las personas diabéticas y obesas logran la euglucemia después de la cirugía. Esto se debe a que la restricción del volumen gástrico conlleva a una saciedad temprana, menor ingesta de comida; por lo tanto, reduce la ingesta calórica. Mejorando de esta manera la sensibilidad a la insulina y por ende, una disminución de los niveles de glucosa en ayunas ${ }^{(34)}$.

Además, el aumento del GLP-1 también contribuye al aumento de la sensibilidad hacia la insulina. Puesto que, el GLP-1 tiene muchas funciones fisiológicas, entre ellas, la estimulación de la secreción de insulina por el páncreas y un aumento de la sensibilidad a la insulina. Por esto, el GLP-1 también es considerado un factor indispensable en la rápida resolución de la Diabetes mellitus, al disminuir la insulinorresistencia ${ }^{(8,35)}$

\section{- Disminución de la inflamación}

En personas obesas cuyo organismo se encuentra en un estado inflamatorio, la pérdida de peso postquirúrgica, es un factor determinante para reducir el nivel de marcadores proinflamatorios al tener un efecto anti-inflamatorio ${ }^{(36)}$.

En primer lugar, la disminución de la ingesta calórica, origina cambios en la expresión génica de citocinas que reducen la IL-6 y TNF-a en la grasa abdominal y aumenta la expresión de IL-10, una citoquina antiinflamatoria ${ }^{(36)}$. Así mismo, la pérdida de peso y el aumento de GLP-1 aumenta la expresión de la adiponectina, la cual es una adipocina con efecto antiinflamatorio ${ }^{(36)}$. Además, se ha demostrado que la pérdida de peso después de la cirugía bariátrica reduce el estrés oxidativo y aumenta la actividad de la enzima antioxidante, como consecuencia hay una modulación de ROS que reduce la secreción de citocinas ${ }^{(36,37)}$

La inflamación sistémica en la obesidad, desempeña un papel de gravedad frente al COVID-19, las personas obesas tienden a requerir una estancia en la unidad de cuidados intensivos (UCl) y tienen mayores tasas de mortalidad. La pérdida de peso posquirúrgica disminuye el estado inflamatorio y por ende contribuye a una menor gravedad en el desarrollo de la enfermedad ${ }^{(38)}$.

\section{- $\quad$ Aumento de la inmunidad}

La pérdida de peso inducida por la cirugía bariátrica conlleva a mecanismos involucrados en los cambios en la inmunidad adaptativa y celular. Por ejemplo, se ha evidenciado que después de BGYR los linfocitos B cambian de fenotipo de efector a regulador, suprimiendo la secreción de citocinas proinflamatorias por los linfocitos Tfh. Además de una reducción en el recuento de linfocitos $B, T C D 8+y$ asesinos naturales (NK). Así mismo, un aumento en la subpoblación de linfocitos antiinflamatorios asociado con la disminución de los niveles de glucosa después de la cirugía bariátrica ${ }^{(37)}$.

La disminución de la inmunidad en pacientes obesos, predisponen a una infección severa por COVID-19. La pérdida de peso postquirúrgica disminuye la inflamación, contribuyendo al aumento de la función de las células inmunes ${ }^{(37)}$. 


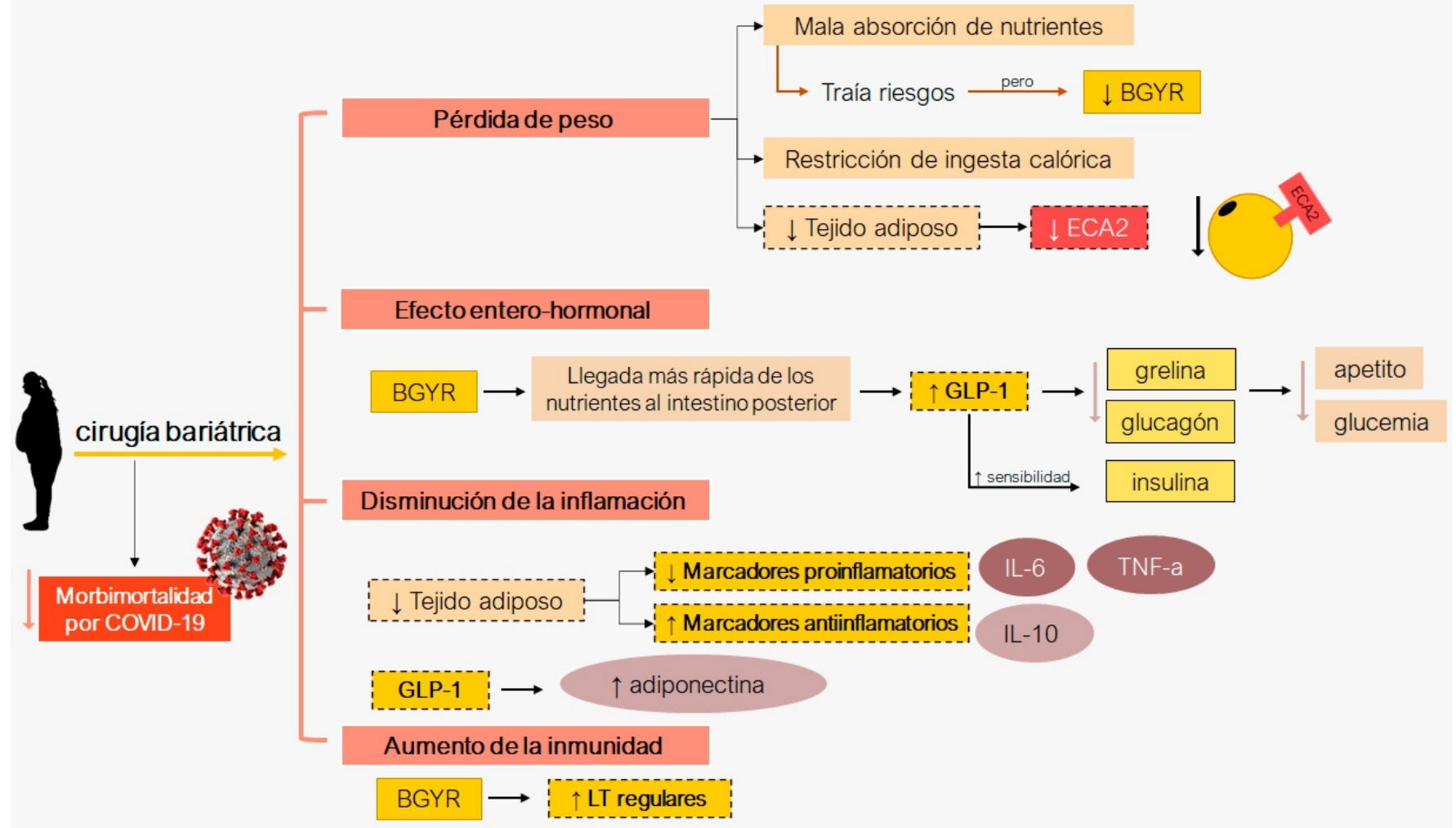

BGYR: bypass gástrico con técnica de Y de Roux, GLP-1: Péptido similar al glucagón tipo 1, IL: Interleucinas, TNF: Factor de necrosis tumoral, ECA: Enzima convertidora de angiotensina, LT: Linfocito T.

Figura 2.

Fisiopatología de la cirugía bariátrica en Covid-19.

\section{OPINIÓN DE EXPERTOS}

Una encuesta realizada a cirujanos bariátricos y no bariátricos de Reino Unido, mostró una discrepancia en cuanto a sus opiniones sobre este tipo de cirugías en el contexto de la pandemia por COVID-19 ${ }^{(39)}$.

Los cirujanos no bariátricos clasificaron a la cirugía bariátrica como de baja prioridad sin necesidad de cambiar los criterios de puesta en servicio, mientras que la mayoría de los cirujanos bariátricos valoraban la cirugía bariátrica como una prioridad media a alta con la necesidad de cambiar los criterios de puesta en servicio para facilitar el acceso. Muchos de ellos consideran la cirugía bariátrica como procedimientos electivos no urgentes que no tienen la máxima prioridad en comparación con otros tipos de intervención quirúrgica como: la cirugía de hernias, cirugía de mama, traumatología, cirugía de emergencia y oncología quirúrgica. De acuerdo con esta perspectiva, un paciente con cáncer debe tener prioridad para prevenir la diseminación, todo esto conduce a la idea de prioridad en intervenciones quirúrgicas. Sin embargo, esta puede no ser precisa, ya que los pacientes indicados para cirugía bariátrica también tienen un alto riesgo de desarrollar morbilidades graves, como las que se han mencionado en párrafos anteriores, además de estar en el grupo de alto riesgo de COVID-19 grave / crítico $^{(39)}$

Otra encuesta realizada a cirujanos de Argentina concluyó en que se requieren ciertas condiciones para reiniciar las cirugías bariátricas electivas, y de estas, la recomendación de la sociedad bariátrica fue la más esperada. Por otro lado, los resultados de esta encuesta permitieron elaborar el protocolo de manejo en este país en el contexto de la pandemia por COVID19, a través de la cual, la selección de pacientes se centró en evitar la operación de pacientes positivos para COVID-19 asintomáticos, seguida de la exclusión de los individuos con mayor riesgo de enfermedad viral debido a la edad, enfermedad pulmonar crónica o inmunosupresión ${ }^{(40)}$.

\section{CONCLUSIONES}

- La obesidad genera cambios en la ventilación pulmonar y está asociada a comorbilidades como la HTA, diabetes mellitus tipo 2, entre otras, todas estas condiciones convierten a las personas obesas en pacientes más susceptibles a complicaciones por esta enfermedad, lo que conduce a un cuadro grave y posterior desenlace fatal.

- A pesar de ser considerada por algunos médicos, una cirugía electiva no urgente, la cirugía bariátrica constituye una medida a largo plazo que logra la reducción del estado inflamatorio generado por el aumento de grasa corporal, lo que disminuye el riesgo de complicaciones en la COVID- 19.

- Una persona con obesidad al someterse a una cirugía 
bariátrica, puede llegar a perder peso, esto disminuirá el riesgo de tener una forma severa de COVID-19 de forma significativa, así mismo disminuirá la mortalidad por COVID-19.

La cirugía bariátrica RYGB, disminuye la resistencia a la insulina, favorece mayor pérdida de peso y la resolución de la inflamación como la inmunodepresión en pacientes con obesidad mórbida y/o sus comorbilidades asociadas; disminuyendo el riesgo de la enfermedad COVID-19.

\section{RECOMENDACIONES}

- Manejo y control de la obesidad con prácticas saludables que incluyen la alimentación saludable y el ejercicio físico constante. Lo cual, ayuda a la reducción del estado pro inflamatorio provocado por la obesidad.

Cribado obligatorio antes de la cirugía bariátrica para los pacientes con obesidad y/o comorbilidades asociadas dados los riesgos de complicaciones graves por COVID-19.

El abordaje laparoscópico es favorable para los pacientes y debe preferirse al uso de técnicas abiertas en la pandemia, debido al riesgo de contagio.

\section{REFERENCIAS BIBLIOGRÁFICAS}

1. Organización Mundial de la Salud. Obesidad y sobrepeso [Internet]. Who.int. 2021 [acceso: 04/04/21]. Disponible en: https://www.who.int/es/news-room/fact-sheets/detail/obesityand-overweight

2. Boletín epidemiológico del Perú 2020 [Internet]. Dge.gob.pe. 2021 [ a c c e s o: $05 / 04 / 21$ ]. D is p o n i ble e n : https://www.dge.gob.pe/epipublic/uploads/boletin/boletin_2020 39.pdf

3. INEI - Perú: Enfermedades No Transmisibles y Transmisibles, 2020 [Internet]. INEI.go.pe. 2021 [cited 12 December 2021]. Disponible h https://www.inei.gob.pe/media/MenuRecursivo/publicaciones_dig itales/Est/Lib1796/

4. Stefan N, Birkenfeld A, Schulze M, Ludwig D. Obesity and impaired metabolic health in patients with COVID-19. Nat Rev Endocrinol. 2020;16(7):341-342. DOI: 10.1038/s41574-020-0364-6.

5. Fakharian A, Moghadassi $H$, Vasheghani M, Moghadasi A. Bariatric Surgery During the COVID-19 Pandemic-the Perspective of Physicians and Patients. Obesity surgery.2021; 31(3): 1339-1341. DOI: 10.1007/s11695-020-04998-z.

6. Santos M, Mackey E, Gaffka B, Ward W, Burton E. Bariatric and metabolic surgery during and after the COVID-19 pandemic. The Lancet Diabetes \& Endocrinology. 2020; 8(9): 742-743. DOI:10.1016/s2213-8587(20)30278-3.

7. Sudlow A, le Roux C, Pournaras D. The metabolic benefits of different bariatric operations: what procedure to choose?. Endocrine connections. 2020; 9(2): 28-35. DOI: 10.1530/EC-19-0467.

8. Peterson K, Anderson J, Boundy E, Ferguson L, Erickson K. Rapid Evidence Review of Bariatric Surgery in Super Obesity (BMI $\geq 50$ $\mathrm{kg} / \mathrm{m} 2$ ). Journal of general internal medicine. 2017; 32(1): 56-64. DOI 10.1007/s11606-016-3950-5.

9. Hussain A, Mahawar K, Xia Z, Yang W, El-Hasani S. Obesity and mortality of COVID-19. Meta-analysis. Obes Res Clin Pract. 2020;14(4):295-300. DOI:10.1016/j.orcp.2020.07.002.

10. Marseglia L, Manti S, D'Angelo G, Nicotera A, Parisi E, Di Rosa G, et al. Oxidative stress in obesity: a critical component in human diseases. Int J Mol Sci. 2014;16(1):378-400. DOI: 10.3390/ijms16010378.

11. Wang B \& Trayhurn P. Acute and prolonged effects of TNF-alpha on the expression and secretion of inflammation-related adipokines by human adipocytes differentiated in culture. Pflüg Arco. 2006; 452(4):418-427. DOI: 10.1007/s00424-006-0055-8.

12. Fischer $P \&$ Möller $P$. The immune system of adipose tissue: obesityassociated inflammation. Pathologe. 2020;41(3):224-229. DOI:
$10.1007 / \mathrm{s} 00292-020-00782-z$

13. Muñoz M, Mazure R, Culebras J. Obesidad y sistema inmune. Nutr Hosp [Internet]. 2004; 19 (6): 319-324.

14. Andersen C, Murphy K, Fernandez M. Impact of Obesity and Metabolic Syndrome on Immunity. Adv Nutr. 2016;7(1):66-75. DOI: 10.3945/an.115.010207.

15. Sánchez R, Garcia A, Breton I, Lecube A, Moizé V, Arroyo J, et al. Obesity and SARS-CoV-2: Considerations on bariatric surgery and recommendations for the start of surgical activity. Cir Esp. 2021; 99(1): 4-10. DOI: 10.1016/j.cireng.2020.12.015.

16. Bel P, Poitou C, Genser L, Marchelli F, Aron J, Ciangura C, et al. COVID19 and its severity in bariatric surgery operated patients. The Obesity Society. 2020; 29(1): 24-28. DOI: 10.1002/oby.23026.

17. Dixon A \& Peters U. The effect of obesity on lung function. Expert review of respiratory medicine. 2018; 12(9): 755-767. DOI: 10.1080/17476348.2018.1506331.

18. Albashir A. The potential impacts of obesity on COVID-19. Clinical medicine. 2020; 20(4): 109-113. DOI: 10.7861/clinmed.2020-0239.

19. Petrilli C, Jones S, Yang J, Rajagopalan H, O'Donnell L, Chernyak Y, et al. Factors associated with hospitalization and critical illness among 4,103 patients with COVID-19 disease in New York city. medRxiv. 2020 [acceso: 28/03/21]. DOI: 10.1101/2020.04.08.20057794.

20. Docherty A, Harrison E, Green C, Hardwick H, Pius R, Norman L, et al. Features of 16,749 hospitalised UK patients with COVID-19 using the ISARIC WHO clinical characterisation protocol. medRxiv. 2020 [acceso: 20/03/21]. DOI: 10.1101/2020.04.23.20076042.

21. Herrera R \& Lesmes B. Obesity in the COVID era: A global health challenge. Endocrinol Diabetes Nutr. 2021 ;68(2):123-129. DOI: 10.1016/j.endinu.2020.10.001.

22. Ratneswaran C., Murphy P., Hart N., Steier J. Obesity, Respiratory Mechanics and Its Impact on the Work of Breathing, Neural Respiratory Drive, Gas Exchange and the Development of SleepDisordered Breathing. Mechanical Ventilation in the Critically III Obese Patient. Springer. 2018: 15-25. DOI: 10.1007/978-3-319-492537_2

23. Poma EG, Guerra HG. La obesidad como factor de riesgo para COVID19. Centro de Investigación de Bioquímica y Nutrición. Instituto de Investigación FMH - USMP. 2020

24. Laffin M \& Karmali S. An Update on Bariatric Surgery. Current Obesity Reports. 2014; 3(3): 316-320. DOI:10.1007/s13679-014-0111-1.

25. Marchesi F, Valente M, Riccò M, Rottoli M, Baldini E, Mecheri F, et al. Effects of Bariatric Surgery on COVID-19: a Multicentric Study from a High Incidence Area. Obes Surg. 2021 Jun;31(6):2477-2488. doi: 10.1007/s11695-020-05193-w. Epub 2021 Jan 8. PMID: 33417099; PMCID: PMC7791147.

26. Aminian A, Fathalizadeh A, Tu C, Scott W, Pantalone K, Griebeler M, et al. Association of prior metabolic and bariatric surgery with severity of coronavirus disease 2019 (COVID-19) in patients with obesity. Surgery for Obesity and Related Diseases. 2021;17(1): 208-214. DOI: 10.1016/j.soard.2020.10.026.

27. Aminian A \& Tu C. Association of Bariatric Surgery with Clinical Outcomes of SARS-CoV-2 Infection: a Systematic Review and Metaanalysis in the Initial Phase of COVID-19 Pandemic. OBES SURG [Internet]. 2021 [acceso: 01/04/21]. DOI: 10.1007/s11695-02005213-9.

28. Landecho $M$, \& Frühbeck $G$. Benefits of bariatric surgery prior to SARS CoV 2 infection in modulating the response to COVID-19. Obesity. 2020; 29(1): 19. DOI:10.1002/oby.23059.

29. Prior Bariatric Surgery Lessens the Severity of SARS-CoV-2 Infection [Internet]. Consult QD. 2021 [acceso: 15/03/21]. Disponible en: https: / / consultqd.clevelandclinic.org/prior-bariatric-surgerylessens-the-severity-of-sars-cov-2-infection/

30. Uccelli M, Cesana G, De Carli S, Ciccarese F, Oldani A, Zanoni A\& et al. COVID-19 y obesidad : ¿Es protectora la cirugía bariátrica? Análisis retrospectivo de 2.145 pacientes sometidos a cirugía bariátricametabólica en un centro de alto volumen en Italia (Lombardía). Obes Surg. 2021; 31 (3): 942-948. DOI: 10.1007 / s11695-020-05085-z.

31. Singhal R, Tahrani A, Ludwig C, Mahawar K; GENEVA collaborators. Global 30-day outcomes after bariatric surgery during the COVID-19 pandemic (GENEVA): an international cohort study. Lancet Diabetes Endocrinol. 2021;9(1):7-9. DOI: 10.1016/S2213-8587(20)30375-2.

32. Rubino F, Cohen R, Mingrone G, le Roux C, Mechanick J, Arterburn D, et al. Bariatric and metabolic surgery during and after the COVID-19 pandemic: DSS recommendations for management of surgical candidates and postoperative patients and prioritisation of access to surgery. The Lancet Diabetes \& Endocrinology. 2020; 8(7): 640-648. 
DOI:10.1016/s2213-8587(20)30157-1.

33. Zhang Y, Somers K, Becari C, Polonis K, Pfeifer M, et al. Comparative expression of renin-angiotensin pathway proteins in visceral versus subcutaneous fat. Front Physiol. 2018;9:1370. DOI: 10.3389/fphys.2018.01370.

34. Iannelli A, Favre G, Frey S, Esnault V, Gugenheim J, Bouam S, et al. Obesity and COVID-19: ACE 2, the Missing Tile. Obesity surgery. 2020 30(11): 4615-4617. DOI: 10.1007/s11695-020-04734-7. PMID: 32451913.

35. Sethu S \& Reddy K. Bariatric Surgery. Endocrinology and Metabolism Clinics of North America. 2016;45(4): 905-921. DOI:10.1016/j.ecl.2016.06.011.

36. Bianchi V. Weight loss is a critical factor to reduce inflammation. Clinical Nutrition ESPEN. 2018;28:21-35 DOI:10.1016/j.clnesp.2018.08.007.

37. Villarreal J, Cuéllar R, Ramos M, Rubio N, Castillo E, Elizondo L, et al.
Interplay between the Adaptive Immune System and Insulin Resistance in Weight Loss Induced by Bariatric Surgery. Oxidative Medicine and Cellular Longevity. 2019: 1-14. DOI:10.1155/2019/3940739.

38. Yeo C, Ahmed S, Oo A, Koura A, Sanghvi K, Yeo D. COVID-19 and Obesity-the Management of Pre- and Post-bariatric Patients Amidst the COVID-19 Pandemic. Obesity surgery. 2020; 30(9): 3607-3609. DOI: $10.1007 / \mathrm{s} 11695-020-04670-6$.

39. Ghanem A, Sameh E, Cousins J, Kerrigan D, Rashid A. Bariatric surgery during COVID-19 in the UK: a British Obesity and Metabolic Surgery Society (BOMSS) survey. Surgical Endoscopy. 2021:1-11. DOI: 10.1007/s00464-021-08314-3.

40. Axel F, Martinez P, Behrens E, Fiolo F, Ramos A. COVID-19 Pandemic and Bariatric Surgery in Argentina. OBES SURG. 2020; 30(12): 5170-5176. DOI: 10.1007/s11695-020-05004-2. 